\title{
ON A NONLINEAR CONGRUENTIAL PSEUDORANDOM NUMBER GENERATOR
}

\author{
TAKASHI KATO, LI-MING WU, AND NIRO YANAGIHARA
}

\begin{abstract}
A nonlinear congruential pseudorandom number generator with modulus $M=2^{w}$ is proposed, which may be viewed to comprise both linear as well as inversive congruential generators. The condition for it to generate sequences of maximal period length is obtained. It is akin to the inversive one and bears a remarkable resemblance to the latter.
\end{abstract}

\section{INTRODUCTION}

A standard method of generating uniform pseudorandom numbers in the interval $I=[0,1)$ (denoted as PRN) is the linear congruential one, which is given as follows: For a large modulus $M$, let

$$
Z_{M}=\{0,1, \ldots, M-1\}=Z / M .
$$

A sequence $\left\{y_{n}\right\}$ of integers in $Z_{M}$ is generated by the linear recursion

$$
y_{n+1} \equiv c y_{n}+b \quad(\bmod M), \quad n=0,1, \ldots,
$$

where $c, b \in Z_{M}$. The PRN are obtained by the normalization

$$
x_{n}=y_{n} / M .
$$

This linear method is widely used, and has been investigated by several authors [9]. However, there is some drawback owing to the linearity of the recursion, e.g., so-called coarse lattice structure. This state of affairs provided the motivation for several recent proposals of nonlinear congruential generators $[1,5,9,12]$.

Among them, one of the most interesting is the inversive congruential method [12], with prime modulus ( $M=p$ for a prime $p$ ) or power of two modulus $\left(M=2^{w}\right.$ for a large integer $w$ ). The latter is described as follows: For $M=2^{w}$, let

$$
G_{M}=\{1,3, \ldots, M-1\}=\{\text { positive odd integers less than } M\} .
$$

For any $u \in G_{M}$, there is a unique $\bar{u} \in G_{M}$ such that $u \bar{u} \equiv 1(\bmod M)$. Now a sequence $\left\{y_{n}\right\} \subset G_{M}$ is generated by the inversive recursion

$$
y_{n+1} \equiv a \bar{y}_{n}+b \quad(\bmod M), \quad n=0,1, \ldots,
$$

Received by the editor October 7, 1994 and, in revised form, February 12, 1995.

1991 Mathematics Subject Classification. Primary 65C10; Secondary 11K45.

Key words and phrases. Pseudorandom number, maximal period length, nonlinear congruential generator, power of two modulus.

(C)1996 American Mathematical Society 
in which $a, b \in Z_{M}$ are chosen so that $y_{n} \in G_{M}$ implies $y_{n+1} \in G_{M}$.

In the present note, we propose another nonlinear method similar to (1.3), i.e., for the modulus $M=2^{w}$, we put with $y_{0} \in G_{M}$,

$$
y_{n+1} \equiv a \bar{y}_{n}+b+c y_{n} \quad(\bmod M), \quad n=0,1, \ldots
$$

in which of course $a, b, c \in Z_{M}$ should be chosen so that $y_{n} \in G_{M}$ implies $y_{n+1} \in$ $G_{M}$. The PRN $\left\{x_{n}\right\}$ is defined by (1.2).

We will show that the modified inversive method (1.4) bears a close resemblance to (1.3). That is, we prove the following theorem.

Theorem. Let $M=2^{w}, w \geq 3$. Then the PRN $\left\{x_{n}\right\}$ derived from (1.4) is purely periodic with period $M / 2$ if and only if

$$
a+c \equiv 1(\bmod 4) \quad \text { and } \quad b \equiv 2(\bmod 4) .
$$

Among the constants in the theorem, one of $a$ or $c$ may be zero, hence (1.4) can be viewed as to comprise both (1.1) and (1.3).

The discrepancy as well as lattice structure of the sequence $\left\{x_{n}\right\}$, generated by (1.4), will be studied in future papers.

Our proof of the theorem is very similar to the proofs in [5, Theorem] and [12, Theorem 8.9]. But we hope that the modified method (1.4) would be of some interest. By the way, we note that the difference equation

$$
y(t+1)=y(t)+b+a / y(t)
$$

has been studied from the viewpoint of complex analytic theory $[7,8,13,14,15]$. Its solutions exhibit distinctly fractal features.

\section{Proof of Theorem}

We divide the proof into three subsections (I),(II),(III).

(I) Necessity. Write the period of $\left\{x_{n}\right\}$ as $\operatorname{per}\left(x_{n}\right)$. Obviously, $\operatorname{per}\left(x_{n}\right) \leq M / 2$.

Suppose that $\left\{x_{n}\right\}$ is purely periodic with $\operatorname{per}\left(x_{n}\right)=M / 2$. Then $\left\{y_{0}, y_{1}, \ldots\right.$, $\left.y_{M / 2-1}\right\}=G_{M}$, so we can assume that $y_{0}=1$. If we consider the sequence $\left\{y_{n}\right\}$ modulo 4 , then it has period 2 ; hence $y_{2} \equiv 1(\bmod 4)$. If this sequence is taken modulo 8 , then it has period 4 ; hence $y_{2} \neq 1(\bmod 8)$, and so $y_{2} \equiv 5(\bmod 8)$. Since $\bar{u} \equiv u(\bmod 8)$ for $u \in G_{M}$, it follows from (1.4) that

$$
y_{2} \equiv c(a+b+c)+b+a(a+b+c)=(a+c)^{2}+(a+c+1) b \bmod 8 .
$$

Suppose $a+c$ is even. Then $b$ must be odd since $y_{1} \equiv a+b+c(\bmod 8) \in G_{M}$. Put $a+c=2 r, b=1+2 s$. Then $y_{2}=4 r^{2}+(1+2 r)(1+2 s)=1+2(r+s)+4 r s+4 r^{2}$, which must be $\equiv 1(\bmod 4)$. Hence, $r+s=2 t, t \in Z$. Then $y_{1}=1+4 t \equiv 1(\bmod$ $4)$, which contradicts that $\left\{y_{n}\right\}$ has period $2(\bmod 4)$. Therefore, $a+c$ must be odd. Hence, $(a+c)^{2} \equiv 1(\bmod 8)$, and we have

$$
y_{2} \equiv 1+(a+c+1) b \quad(\bmod 8),
$$

so $(a+c+1) b \equiv 4(\bmod 8)$. This implies $a+c \equiv 1(\bmod 4), b \equiv 2(\bmod 4)$. 
(II) Sufficiency for the case where $c$ is an even number. Suppose $a+c \equiv 1$ $(\bmod 4)$ and $b \equiv 2(\bmod 4)$. Consider first the case $y_{0}=1$. For $M=8$, it is checked by the above arguments that $\operatorname{per}\left(y_{n}\right)=4$. Now let $M=2^{w}$ with $w \geq 4$.

In this subsection we suppose that $c$ is an even number.

Define a sequence $\left\{\alpha_{n}\right\} \subset G_{M}, n=0,1,2, \ldots$, by

$$
\alpha_{n+2} \equiv \bar{\alpha}_{n}\left(a \alpha_{n}^{2}+b \alpha_{n} \alpha_{n+1}+c \alpha_{n+1}^{2}\right)(\bmod M) .
$$

Put $\alpha_{0}=\alpha_{1}=1$. By induction on $n$, we obtain

$$
y_{n} \equiv \bar{\alpha}_{n} \alpha_{n+1} \quad(\bmod M), n=0,1, \ldots
$$

Write (2.1) as

$$
\begin{gathered}
\alpha_{n+2} \equiv(a+c) \alpha_{n}+b \alpha_{n+1}+c \beta_{n}(\bmod M), \\
\beta_{n}=\bar{\alpha}_{n}\left(\alpha_{n+1}{ }^{2}-\alpha_{n}{ }^{2}\right) .
\end{gathered}
$$

With the integer matrix

$$
A=\left(\begin{array}{cc}
0 & 1 \\
a+c & b
\end{array}\right)
$$

we see, from (2.3), that

$$
\left(\begin{array}{c}
\alpha_{n+1} \\
\alpha_{n+2}
\end{array}\right) \equiv A\left(\begin{array}{c}
\alpha_{n} \\
\alpha_{n+1}
\end{array}\right)+c\left(\begin{array}{c}
0 \\
\beta_{n}
\end{array}\right)
$$

and so

$$
\left(\begin{array}{c}
\alpha_{n} \\
\alpha_{n+1}
\end{array}\right) \equiv A^{n}\left(\begin{array}{c}
\alpha_{0} \\
\alpha_{1}
\end{array}\right)+R_{n}(\bmod M)
$$

where

$$
R_{n}=c\left(\begin{array}{c}
0 \\
\beta_{n-1}
\end{array}\right)+c A\left(\begin{array}{c}
0 \\
\beta_{n-2}
\end{array}\right)+\cdots+c A^{n-1}\left(\begin{array}{c}
0 \\
\beta_{0}
\end{array}\right) .
$$

By induction on $h \geq 2$, using $a+c \equiv 1(\bmod 4), b \equiv 2(\bmod 4)$, it is shown, as in [12, p.188], that for $m=2^{h}$

$$
A^{m} \equiv\left(\begin{array}{cc}
2 m p+m+1 & 2 m q+3 m \\
2 m q+3 m & 2 m p+3 m+1
\end{array}\right) \quad \bmod 4 m=2^{h+2},
$$

for all $h \geq 2$, with some integers $p, q$.

We will show that, for $m=2^{h}(2 \leq h \leq w-1)$,

$$
R_{m}=4 m S_{m}
$$

for an integer vector $S_{m}$. Equation (2.6) holds for $h=2$, since $c$ is assumed to be even. Now suppose it holds for $m=2^{h}$. Then

$$
\alpha_{m}=1+2 m(p+q)+4 m+4 m T_{0}+4 m S_{m}^{(0)}+s_{m} M,
$$




$$
\alpha_{m+1}=1+2 m(p+q)+6 m+4 m T_{1}+4 m S_{m}^{(1)}+s_{m+1} M
$$

with integers $T_{0}, T_{1}, s_{m}, s_{m+1}$, where we write $S_{m}={ }^{t}\left(S_{m}{ }^{(0)}, S_{m}{ }^{(1)}\right)$. Hence, we have

$$
\alpha_{m}=\alpha_{0}+2 m U_{0}, \quad \alpha_{m+1}=\alpha_{1}+2 m U_{1}
$$

with integers $U_{0}, U_{1}$. Then

$$
\beta_{m}=\bar{\alpha}_{m}\left\{\left(1+2 m U_{0}\right)^{2}-\left(1+2 m U_{1}\right)^{2}\right\}=4 m W_{0}=\beta_{0}+4 m W_{0}
$$

for an integer $W_{0}$. Thus,

$$
\alpha_{m+2}=(a+c)\left(\alpha_{0}+2 m U_{0}\right)+b\left(\alpha_{1}+2 m U_{1}\right)+c \beta_{m}=\alpha_{2}+2 m U_{2},
$$

with an integer $U_{2}$. It is easy to see that

$$
\bar{\alpha}_{m+1}=\bar{\alpha}_{1}+2 m V_{1}, \quad \bar{\alpha}_{m+2}=\bar{\alpha}_{2}+2 m V_{2} .
$$

Then

$$
\begin{aligned}
\beta_{m+1}= & \bar{\alpha}_{m+1}\left\{\left(\alpha_{2}+2 m U_{2}\right)^{2}-\left(\alpha_{1}+2 m U_{1}\right)^{2}\right\} \\
= & 4 m \bar{\alpha}_{m+1}\left(\alpha_{2} U_{2}-\alpha_{1} U_{1}+m U_{2}{ }^{2}-m U_{1}^{2}\right) \\
& \quad+\left(\bar{\alpha}_{m+1}-\bar{\alpha}_{1}\right)\left(\alpha_{2}{ }^{2}-\alpha_{1}{ }^{2}\right)+\bar{\alpha}_{1}\left(\alpha_{2}{ }^{2}-\alpha_{1}{ }^{2}\right) \\
= & \beta_{1}+4 m W_{1}
\end{aligned}
$$

with an integer $W_{1}$, since $\alpha_{k+1}^{2}-\alpha_{k}^{2}$ is divided by 8 for any $k$.

Repeating this procedure, we get

$$
\begin{gathered}
\alpha_{m+k}=\alpha_{k}+2 m U_{k} \text { with some integer } U_{k}, \quad k=0, \ldots, m-1, \\
\bar{\alpha}_{m+k}=\bar{\alpha}_{k}+2 m V_{k} \quad \text { with some integer } V_{k}, \quad k=0, \ldots, m-1, \\
\beta_{m+k}=\beta_{k}+4 m W_{k} \quad \text { with some integer } \quad W_{k}, \quad k=0, \ldots, m-1 .
\end{gathered}
$$

Now

$$
\left(\begin{array}{c}
\alpha_{2 m} \\
\alpha_{2 m+1}
\end{array}\right)=A^{2 m}\left(\begin{array}{c}
\alpha_{0} \\
\alpha_{1}
\end{array}\right)+R_{2 m},
$$

in which we obtain by (2.9)

$$
\begin{aligned}
R_{2 m}= & c\left(\begin{array}{c}
0 \\
\beta_{2 m-1}
\end{array}\right)+c A\left(\begin{array}{c}
0 \\
\beta_{2 m-2}
\end{array}\right)+\cdots+c A^{m-1}\left(\begin{array}{c}
0 \\
\beta_{m}
\end{array}\right) \\
& +c A^{m}\left(\begin{array}{c}
0 \\
\beta_{m-1}
\end{array}\right)+c A^{m+1}\left(\begin{array}{c}
0 \\
\beta_{m-2}
\end{array}\right)+\cdots+c A^{2 m-1}\left(\begin{array}{c}
0 \\
\beta_{0}
\end{array}\right) \\
= & c\left(\begin{array}{c}
0 \\
\beta_{m-1}
\end{array}\right)+c A\left(\begin{array}{c}
0 \\
\beta_{m-2}
\end{array}\right)+\cdots+c A^{m-1}\left(\begin{array}{c}
0 \\
\beta_{0}
\end{array}\right) \\
& +A^{m}\left\{c\left(\begin{array}{c}
0 \\
\beta_{m-1}
\end{array}\right)+c A\left(\begin{array}{c}
0 \\
\beta_{m-2}
\end{array}\right)+\cdots+c A^{m-1}\left(\begin{array}{c}
0 \\
\beta_{0}
\end{array}\right)\right\} \\
& +4 m c\left\{\left(\begin{array}{c}
0 \\
W_{m-1}
\end{array}\right)+A\left(\begin{array}{c}
0 \\
W_{m-2}
\end{array}\right)+\cdots+A^{m-1}\left(\begin{array}{c}
0 \\
W_{0}
\end{array}\right)\right\} \\
= & \left.I+A^{m}\right) R_{m}+4 m c W,
\end{aligned}
$$


thus by (2.5) and (2.6) we have, with an integer vector $W$,

$$
R_{2 m}=8 m\left\{\left(I+A^{m}\right) / 2\right\} S_{m}+8 m(c / 2) W=4(2 m) S_{2 m}
$$

since $c$ is even by our assumption. Hence (2.6) holds for $2 m=2^{h+1}$. Thus, we obtain (2.6) for any $m=2^{h}(2 \leq h \leq w-1)$, from which we see by the above arguments that (2.7) holds for any $m=2^{h}(2 \leq h \leq w-1)$.

By (2.7) with $m=M / 2 \quad(h=w-1)$, we obtain $\alpha_{n+M / 2}=\alpha_{n}$ for any $n$, which implies $y_{n+M / 2}=y_{n}$. Therefore, $\operatorname{per}\left(y_{n}\right)$ divides $M / 2$. Since we already know that $\operatorname{per}\left(y_{n}\right) \leq M / 2$, to prove that $\operatorname{per}\left(y_{n}\right)=M / 2$, it suffices to show that $\operatorname{per}\left(y_{n}\right)>M / 4$.

If we had $\operatorname{per}\left(y_{n}\right) \leq M / 4$, then $y_{M / 4}=y_{0}=1$, and so $\alpha_{M / 4+1} \equiv \alpha_{M / 4}(\bmod M)$ by (2.2). However, by (2.4) with $n=M / 4$, and by (2.5), (2.6) with $h=w-2$, we obtain a contradiction $\alpha_{M / 4+1} \equiv \alpha_{M / 4}+M / 2(\bmod M)$. So $\operatorname{per}\left(y_{n}\right)=M / 2$ is proved if $y_{0}=1$. In particular, $\left\{y_{0}, y_{1}, \ldots, y_{M / 2-1}\right\}=G_{M}$. If we have an arbitrary initial value $y_{0} \in G_{M}$, then the sequence $y_{0}, y_{1}, \ldots$ is a shifted version of the sequence with initial value 1 , and so again $\operatorname{per}\left(x_{n}\right)=\operatorname{per}\left(y_{n}\right)=M / 2$.

(III) Sufficiency for the case where $c$ is an odd number. Now we turn to the case when $c$ is odd. Then $a$ must be even.

The equation (1.4) can be written as

$$
y_{n+1} \equiv(a+c) y_{n}+b+a \bar{y}_{n}\left(1-y_{n}^{2}\right) \bmod M,
$$

i.e.,

$$
\left(\begin{array}{c}
y_{n+1} \\
1
\end{array}\right) \equiv\left(\begin{array}{cc}
a+c & b \\
0 & 1
\end{array}\right)\left(\begin{array}{c}
y_{n} \\
1
\end{array}\right)+a\left(\begin{array}{c}
z_{n} \\
0
\end{array}\right) \bmod M
$$

in which $z_{n}=\bar{y}_{n}\left(1-y_{n}{ }^{2}\right)$. Put

$$
A=\left(\begin{array}{cc}
a+c & b \\
0 & 1
\end{array}\right)
$$

We obtain that

$$
\left(\begin{array}{c}
y_{n} \\
1
\end{array}\right) \equiv A^{n}\left(\begin{array}{c}
y_{0} \\
1
\end{array}\right)+R_{n}
$$

where

$$
R_{n}=a\left(\begin{array}{c}
z_{n-1} \\
0
\end{array}\right)+a A\left(\begin{array}{c}
z_{n-2} \\
0
\end{array}\right)+\cdots+a A^{n-1}\left(\begin{array}{c}
z_{0} \\
0
\end{array}\right)
$$

Since $a+c \equiv 1(\bmod 4)$ and $b \equiv 2(\bmod 4)$, we see by induction that, for $m=2^{h}, h \geq 1$,

$$
A^{m}=\left(\begin{array}{cc}
1+4 m P_{m} & 2 m+4 m Q_{m} \\
0 & 1
\end{array}\right)
$$

with integers $P_{m}$ and $Q_{m}$. We will show that, for $m=2^{h}, 1 \leq h \leq w-1$,

$$
R_{m}=4 m S_{m}
$$


with integer vector $S_{m}={ }^{t}\left(s_{m}, 0\right)$. Equation (2.13) is easily seen to hold for $m=$ $2(h=1)$. Suppose it holds for $m=2^{h}$. Then

$$
y_{m}=y_{0}+4 m P_{m} y_{0}+2 m+4 m Q_{m}+4 m s_{m}=y_{0}+2 m U_{0}
$$

with an integer $U_{0}$. Further, it is easy to see that

$$
\bar{y}_{m}=\bar{y}_{0}+2 m V_{0}
$$

with an integer $V_{0}$. Then

$$
z_{m}=\left(\bar{y}_{0}+2 m V_{0}\right)\left\{1-\left(y_{0}+2 m U_{0}\right)^{2}\right\}=z_{0}+4 m W_{0}
$$

with an integer $W_{0}$, since $z_{0}=0$. Then

$$
\begin{aligned}
y_{m+1} & \equiv(a+c)\left(y_{0}+2 m U_{0}\right)+b+a\left(z_{0}+4 m W_{0}\right) \\
& =(a+c) y_{0}+b+a z_{0}+2 m U_{1}=y_{1}+2 m U_{1} .
\end{aligned}
$$

Repeating this procedure, we get, for $k=0,1, \ldots, m-1$,

$$
\begin{aligned}
& y_{m+k}=y_{k}+2 m U_{k}, \\
& \bar{y}_{m+k}=\bar{y}_{k}+2 m V_{k}, \\
& z_{m+k}=z_{k}+4 m W_{k} .
\end{aligned}
$$

Thus, with some integer vector $T_{m}$,

$$
\begin{aligned}
R_{2 m}= & a\left(\begin{array}{c}
z_{2 m-1} \\
0
\end{array}\right)+a A\left(\begin{array}{c}
z_{2 m-2} \\
0
\end{array}\right)+\ldots+a A_{m-1}\left(\begin{array}{c}
z_{m} \\
0
\end{array}\right) \\
& +a A^{m}\left(\begin{array}{c}
z_{m-1} \\
0
\end{array}\right)+a A^{m+1}\left(\begin{array}{c}
z_{m-2} \\
0
\end{array}\right)+\ldots+a A^{2 m-1}\left(\begin{array}{c}
z_{0} \\
0
\end{array}\right) \\
= & a\left(\begin{array}{c}
z_{m-1} \\
0
\end{array}\right)+a A\left(\begin{array}{c}
z_{m-2} \\
0
\end{array}\right)+\ldots+a A^{m-1}\left(\begin{array}{c}
z_{0} \\
0
\end{array}\right) \\
& +A^{m}\left\{a\left(\begin{array}{c}
z_{m-1} \\
0
\end{array}\right)+a A\left(\begin{array}{c}
z_{m-2} \\
0
\end{array}\right)+\ldots+a A^{m-1}\left(\begin{array}{c}
z_{0} \\
0
\end{array}\right)\right\} \\
& +4 m a\left\{\left(\begin{array}{c}
W_{m-1} \\
0
\end{array}\right)+A\left(\begin{array}{c}
W_{m-2} \\
0
\end{array}\right)+\ldots+A^{m-1}\left(\begin{array}{c}
W_{0} \\
0
\end{array}\right)\right\} \\
= & R_{m}+A^{m} R_{m}+4 m a T_{m} \\
= & 4 \times(2 m)\left[\left(I+A^{m}\right) / 2\right] S_{m}+4 \times(2 m)(a / 2) T_{m} \\
= & 4 \times(2 m) S_{2 m},
\end{aligned}
$$

since $a$ is even, which shows that (2.13) holds for $2 m=2^{h+1}$. Therefore, (2.13) holds for any $m=2^{h}, 1 \leq h \leq w-1$.

Hence, (2.14) holds for $m=M / 2=2^{w-1}$, i.e., we obtain $y_{M / 2}=y_{0}$. Thus, $\operatorname{per}\left(y_{n}\right)$ divides $M / 2$. Suppose $\operatorname{per}\left(y_{n}\right) \leq M / 4$. Then $y_{M / 4}=y_{0}$. But by (2.12) and $(2.13)$, we obtain $y_{M / 4} \equiv y_{0}+M / 2(\bmod M)$, which is absurd. As in (II), we conclude that $\operatorname{per}\left(y_{n}\right)=M / 2$. 


\section{REFERENCES}

1. J. Eichenauer-Herrmann, Inversive congruential pseudorandom numbers avoid the planes, Math. Comp. 56 (1991), 297-301. MR 91k:65021

2. _ Statistical independence of a new class of inversive congruential pseudorandom numbers, Math. Comp. 60 (1993), 375-384. MR 93d:65011

3. $\_$, On generalized inversive congruential pseudorandom numbers, Math. Comp. 63 (1994), 293-299. MR 94k:11088

4. J. Eichenauer-Herrmann, H. Grothe, H. Niederreiter, and A. Topuzoglu, On the lattice structure of a nonlinear generator with modulus $2^{\alpha}$, J. Comput. Appl. Math. 31 (1990), 81-85. MR 91j:65012

5. J. Eichenauer, J. Lehn, and A. Topuzoglu, A nonlinear congruential pseudorandom number generator with power of two modulus, Math. Comp. 51 (1988), 757-759. MR 89i:65007

6. J. Eichenauer-Herrmann and H. Niederreiter, Lower bounds for the discrepancy of inversive congruential pseudorandom numbers with power of two modulus, Math. Comp. 58 (1992), 775-779. MR 92i:65018

7. T. Kimura, On the iteration of analytic functions, Funkcial. Ekvac. 14 (1971), 197-238. MR 46:2019

8. - On meromorphic solutions of the difference equation $y(x+1)=y(x)+1+\lambda / y(x)$, Symposium on Ordinary Differential Equations, Lecture Notes in Math., vol. 312, SpringerVerlag, Berlin and New York, 1973, pp. 74-86. MR 53:3527

9. D. E. Knuth, The art of computer programming, Vol. 2, Seminumerical Algorithms, 2nd ed., Addison-Wesley, Reading, MA, 1981. MR 83i:68003

10. H. Niederreiter, The serial test for congruential pseudorandom numbers generated by inversions, Math. Comp. 52 (1989), 135-144. MR 90e:65008

11. - Recent trends in random number and random vector generation, Ann. Oper. Res. 31 (1991), 323-345. MR 92h:65010

12. , Random number generation and quasi-Monte Carlo methods, SIAM, Philadelphia, PA, 1992. MR 93h:65008

13. K. Takano, On the hypertranscendency of solutions of a difference equation of Kimura, Funkcial. Ekvac. 16 (1973), 241-254. MR 50:5074

14. N. Yanagihara, Meromorphic solutions of the difference equation $y(x+1)=y(x)+1+\lambda / y(x)$. I, Funkcial. Ekvac. 21 (1978), 97-104. MR 80a:30021

15. - Meromorphic solutions of the difference equation $y(x+1)=y(x)+1+\lambda / y(x)$. II, Funkcial. Ekvac. 21 (1978), 223-240. MR 81h:30028

Department of Mathematics, Faculty of Science, Chiba University, 1-33 Yayoi-cho, INAGE-KU, Chiba City, 263 JAPAN

E-mail address: yanaba@math.s.chiba-u.ac.jp 